\title{
The Chemical Evolution of the Colour Systems Generated by Riccionidin A, 3-Deoxyanthocyanidins and Anthocyanins.
}

\author{
A. Alejo-Armijo, Johan Mendoza, A. Jorge Parola* and Fernando Pina* \\ LAQV, REQUIMTE, Department of Chemistry, College of Sciences and Technology, Universidade \\ NOVA de Lisboa, 2829-516 Caparica, Portugal.
}

\begin{abstract}
The kinetics and thermodynamics (in acidic solutions) of the five chemical species reversibly interconnected by external stimuli, such as $\mathrm{pH}$ and light (multistate) generated by the liverworts colorant riccionidin A were investigated. The degradation products of the multistate formed after 10 days at neutral $\mathrm{pH}$ were identified.

The behaviour of the riccionidin A multistate was compared with previous results reported for the equivalent systems based on 3-deoxyanthocyanidins (found in mosses and ferns) and anthocyanins (ubiquitous in angiosperms). The five chemical species have mutatis mutandis similar structures in the three multistates. The most dramatic difference is the extremely slow interconversion rate between flavylium cation and trans-chalcone in riccionidin A and related compounds multistate (tens of days) when compared with deoxyanthocyanins (a few days) and anthocyanins (several hours) at room temperature. The mole fraction distribution of the five chemical species that constitute the multistate as a function of $\mathrm{pH}$ is also different in the three families of compounds. Some considerations regarding the chemical evolution of the three systems are given.
\end{abstract}

\section{Graphical Abstract}

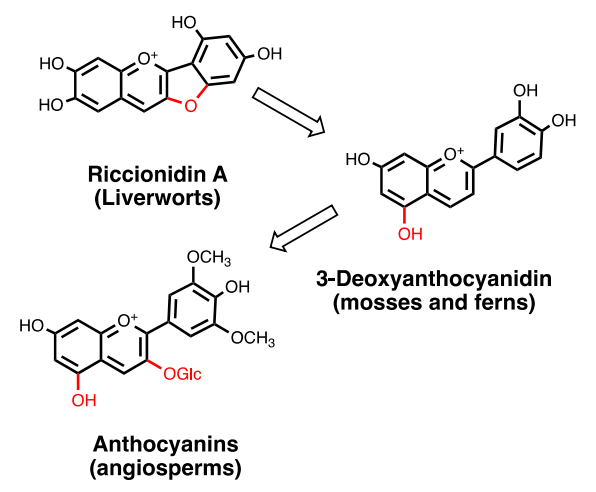

Rates of the inter-conversion between the species of the colour system: Riccionidin A (tens of days), 3-deoxyanthocyanins (a few days) and anthocyanins (several hours). No blue coloured species can be found unless in the anthocyanin's multistate.

Key words: Riccionidin A, auronidins, 3-deoxyanthocyanidins, anthocyanins, kinetics and thermodynamics, chemical evolution. 


\section{Introduction}

Recently, Davies and Andersen ${ }^{1}$ reported on the isolation and characterization of the compound auronidin-2'-neohesperidosideisolated from the liverwort Marchantia polymorpha (marchantia) and proposed the name auronidins for this family of compounds, Scheme 1. Like the aglycones of anthocyanins and 3-deoxyanthocyanins, riccionidin A is the aglycone of auronidin-2'-neohesperidoside, Scheme 1.

The structures shown in Scheme 1 regard only one of the chemical species (appearing under very acidic conditions) of a complex sequence of other compounds reversibly interconnected by external stimuli of $\mathrm{pH}$, light and temperature, see below Fig.1.

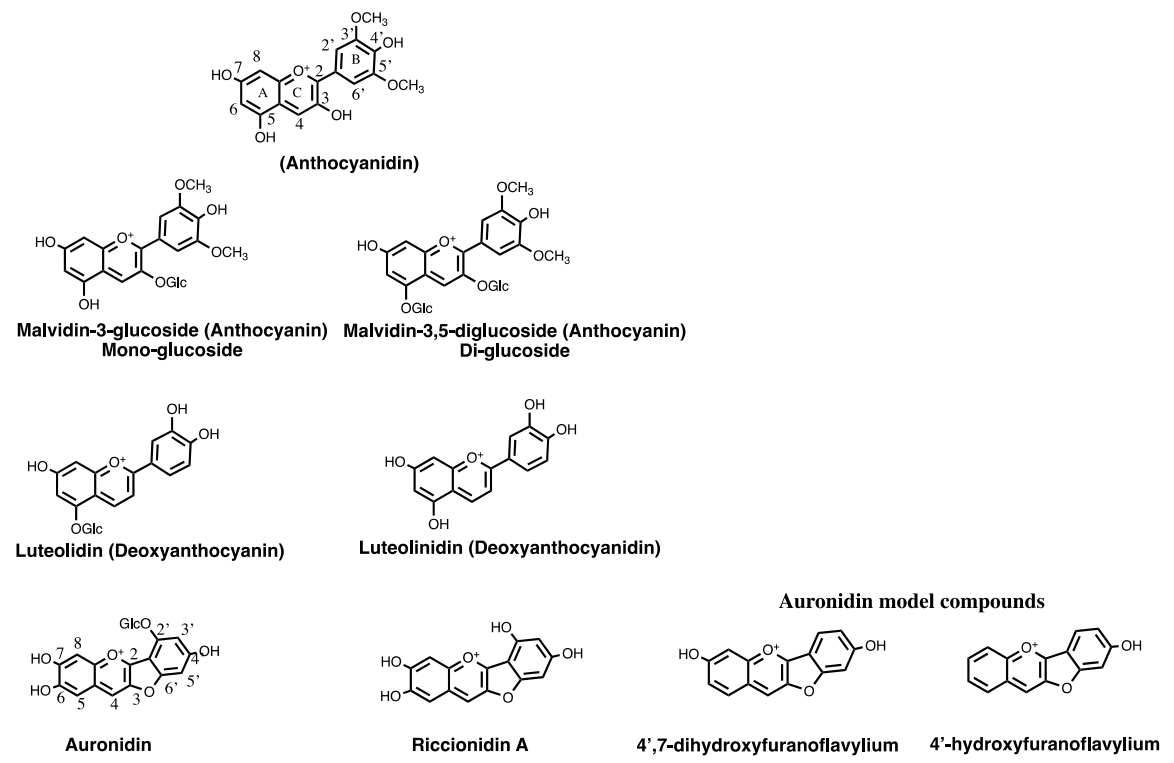

Scheme 1. Anthocyanins, 3-deoxyanthocyanins, auronidin and their respective aglycones together with two riccionidin A synthetic models (Glc=Glucose or other sugars, in the case of the reported auronidin is neohesperidoside). ${ }^{1,}+$

The scope of this work is to investigate the kinetics and thermodynamics of the multistate of riccionidin A (liverworts colorant) and compare with the similar multistates reported for 3-deoxyanthocyanidins (mosses and ferns colorants) and anthocyanins (angiosperms colorants).

\section{State of the Art}

Anthocyanins and deoxyanthocyanidins

\footnotetext{
₹ In reference 1 the first compound is named auronidin-4-neohesperidoside. In the present work, we use auronidin2'-neohesperidoside to be consistent with flavylium derivatives numeration used throughout this work. The riccionidin $\mathrm{A}$ in reference 1 was also considered an auronidin.
} 
Anthocyanins are the molecules that give red to blue colours to many flowers and fruits, but also leaves, vegetables, roots and stems. ${ }^{2}$ The multistate generated by flavylium cations in aqueous solution was firstly investigated for anthocyanins but later was observed that it is also followed by anthocyanidins, deoxyanthocyanidins and related synthetic flavylium salts. ${ }^{3,4,5,6,7}$ More recently it was reported that auronidin model compounds, see Scheme 1, follow in general the same multistate. ${ }^{8,9}$ In acidic to moderately acidic $\mathrm{pH}$ values, five $\mathrm{pH}$ dependent species are reversibly interconverted through four chemical reactions: proton transfer, hydration, tautomerization and cis-trans isomerization, Scheme 2. The anthocyanins multistate of chemical species (from here on designated as multistate) is characterized by its thermodynamics and kinetics. The thermodynamics can be accounted for by the respective energy level diagram, ${ }^{10}$ Fig. 1a, or by a representation of the mole fraction distribution of the multistate species as a function of $\mathrm{pH}$, Fig. $1 \mathrm{~b} .^{4}$

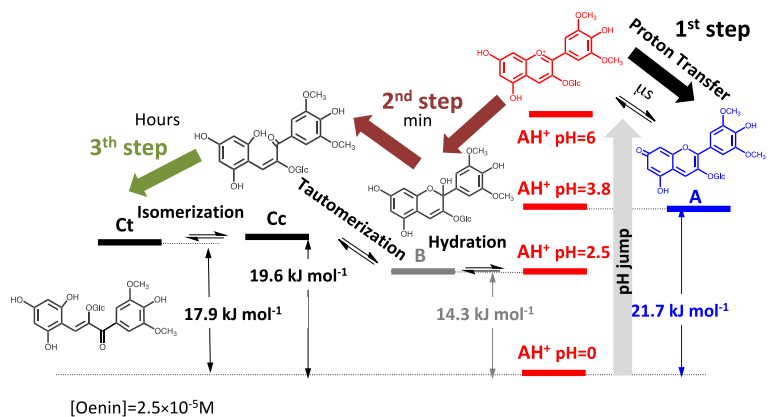

(a)

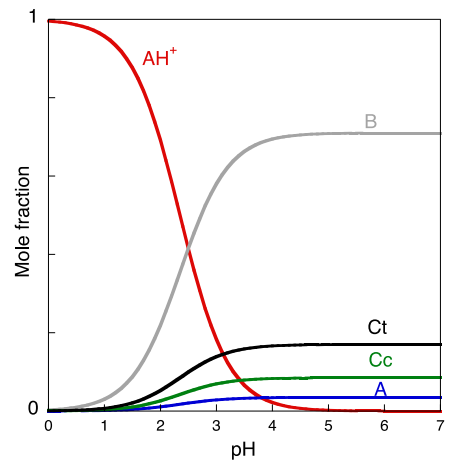

(b)

Figure 1. (a) Thermodynamic energy level diagram of malvidin-3-glucoside (oenin), $2 \times 10^{-5} \mathrm{M}$ showing the three kinetic steps upon a direct $\mathrm{pH}$ jump are shown; (b) mole fraction distribution of the species $\mathrm{AH}^{+}$and the neutral forms $\mathrm{A}, \mathrm{B}, \mathrm{Cc}$ and $\mathrm{Ct}$ at equilibrium.

The kinetics of the anthocyanins multistate is most conveniently studied by addition of base to equilibrated solutions of the flavylium cation at $\mathrm{pH} \leq 1$ (direct $\mathrm{pH}$ jumps) or by addition of acid to equilibrated solutions at higher $\mathrm{pH}$ values back to $\mathrm{pH} \leq 1$ (reverse $\mathrm{pH}$ jumps). Stopped-flow is a very convenient tool to account for the fast kinetic steps. In a direct $\mathrm{pH}$ jump, three distinct kinetic steps take place. The first is proton transfer, eq.(1), that occurs in sub-microseconds, a rate which is faster than the dead time of the stopped-flow, eq.(2). ${ }^{11}$ Considering that the following kinetic steps 
are much slower, the species $\mathbf{A H}^{+}$(red colour) and $\mathbf{A}$ (purple colour) can be considered at equilibrium.

$$
\begin{aligned}
& \mathbf{A H}^{+}+\mathrm{H}_{2} \mathrm{O} \underset{k_{-\mathrm{a}}}{\stackrel{k_{\mathrm{a}}}{\rightleftharpoons}} \mathbf{A}+\mathrm{H}_{3} \mathrm{O}^{+} \quad K_{\mathrm{a}} \quad \text { proton transfer } \\
& k_{1 \text { st(direct) }}=k_{\mathrm{a}}+k_{a}\left[H^{+}\right]
\end{aligned}
$$

The second step corresponds to the disappearance of $\mathbf{A} \mathbf{H}^{+} / \mathbf{A}$ to form the species $\mathbf{B}$ through the hydration reaction followed by a faster tautomerization to give Cc. Since hydration at the $\mathrm{pH}$ values accessed by direct $\mathrm{pH}$ jumps is much slower than tautomerization, the second step is kinetically controlled by the hydration. A particular aspect of the hydration reaction is that it takes place from $\mathbf{A H}^{+}$and not from A (which does not hydrate in acidic to moderately acidic solutions). ${ }^{3}$ This achievement was a breakthrough reported by Brouillard and Dubois and it is fundamental for the comprehension of the multistate. Eq.(3) and eq.(4) together with eq.(5) account for this kinetic step. In eq.(5), $\chi$ represents the mole fraction distribution of $\mathbf{A H}^{+}$and $\mathbf{B}$ at the pseudo-equilibrium, see below.

$$
\begin{array}{lcc}
\mathbf{A H}^{+}+2 \mathrm{H}_{2} \mathrm{O} \underset{k_{\mathrm{h}}}{\stackrel{k_{\mathrm{h}}}{\rightleftharpoons}} \mathbf{B}+\mathrm{H}_{3} \mathrm{O}^{+} & K_{\mathrm{h}} & \text { hydration } \\
\mathbf{B} \underset{k_{-\mathrm{t}}}{\stackrel{k_{\mathrm{t}}}{F}} \mathbf{C c} & K_{\mathrm{t}} \quad \text { tautomerization } \\
k_{2 \text { nd(direct) }}={ }_{A H_{+}} k_{h}+{ }_{B} k_{h}\left[H^{+}\right]=\frac{\left[H^{+}\right]}{\left[H^{+}\right]+K_{a}} k_{h}+\frac{1}{1+K_{t}} k_{h}\left[H^{+}\right]
\end{array}
$$

At this point, a pseudo-equilibrium can be considered, because the last process is by far the slowest and the system evolves to the equilibrium with the species $\mathbf{A H}^{+}, \mathbf{A}, \mathbf{B}$ and $\mathbf{C c}$ having enough time to equilibrate between them.

The last step, controlled by the isomerization, is given by eq.(6) and eq.(7).

$$
\begin{aligned}
& \mathbf{C c} \underset{k_{\mathrm{i}}}{\stackrel{k_{\mathrm{i}}}{\rightleftharpoons}} \mathbf{C t} \\
& k_{3 r d(\text { direct })}={ }_{c c} k_{i}+k_{i}=\frac{K_{h} K_{t}}{\left[H^{+}\right]+K_{a}+K_{h}+K_{h} K_{t}} k_{i}+k_{i}
\end{aligned}
$$




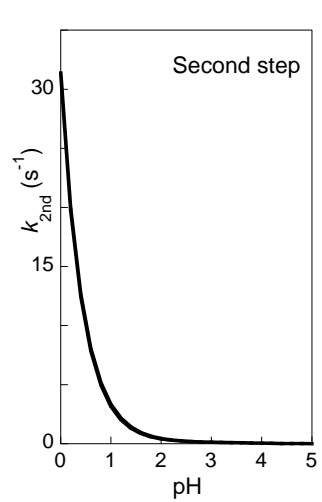

(a)

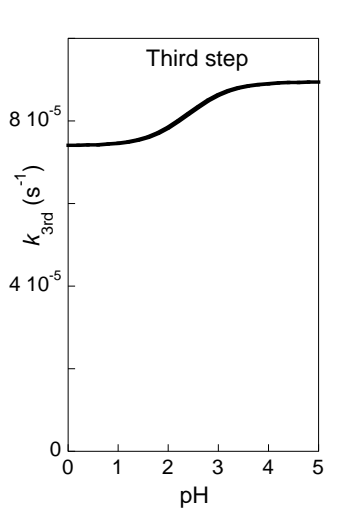

(b)
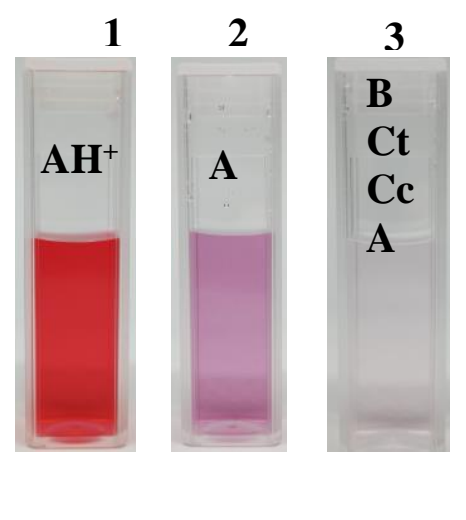

4

$A^{-}$

(c)

Figure 2. (a) Representation of the rate constants versus $\mathrm{pH}$ of the second step for Oenin $2.5 \times 10^{-5} \mathrm{M}$, eq.(5); (b) the same for the third step, which is much slower, eq.(7). The rate and equilibrium constants to represent eq.(5) and eq.(7) were taken from reference [ $\left.{ }^{12}\right]$; (c) colours of Oenin $5.7 \times 10^{-5} \mathrm{M}$ multistate (1) Flavylium cation at $\mathrm{pH}=1.0$; (2) quinoidal base immediately after a direct $\mathrm{pH}$ jump to $\mathrm{pH}=5.0$; (3) at the equilibrium for the same $\mathrm{pH}$ of (2); (4) the intense blue is given by the anionic form of the quinoidal base as shown immediately after a $\mathrm{pH}$ jump to $\mathrm{pH}=8.0$.

Comparison of the ordinate values of Fig. $2 \mathrm{a}$ and Fig. $2 \mathrm{~b}$ confirms that the third step is several orders of magnitude slower in comparison with the second step. The colour of the flavylium cation is red, the quinoidal base is purple and the mono-cationic quinoidal base is blue, Fig. 2c. However, at the equilibrium the main species at higher $\mathrm{pH}$ values is the colourless hemiketal and the fraction of the purple quinoidal base is minor, see Fig. $1 b$ and photo 3 in Fig. 2c.

\section{3-Deoxyanthocyanidins}

3-Deoxyanthocyanidins follow an identical multistate but there are several significant differences regarding the kinetics and the thermodynamics. In 3-deoxyanthocyanidins as well as in many other synthetic flavylium salts there is no cis-trans isomerization barrier and the pseudo-equilibrium does not take place, eq.(8). ${ }^{7}$

$$
\mathrm{A} \underset{\mathrm{H}^{+}}{\stackrel{K_{\mathrm{a}}}{\rightleftharpoons}} \mathrm{AH}^{+} \underset{\mathrm{k}_{\mathrm{h}} \mathrm{H}^{+}}{\stackrel{k_{\mathrm{h}}}{\rightleftharpoons}} \mathrm{B} \stackrel{\text { Equilibrium }}{\stackrel{K_{\mathrm{t}}}{\rightleftharpoons}} \mathrm{Cc} \underset{\mathrm{k}_{-\mathrm{i}}}{\stackrel{k_{\mathrm{i}}}{\rightleftharpoons}} \mathrm{Ct}
$$

This kinetic situation is equivalent to the equilibrium given by eq.(9), with $\mathrm{X}$ and $\mathrm{Y}$ representing respectively the equilibrium between $\mathbf{A} \mathbf{H}^{+}$and $\mathbf{A}$ by one side, $\mathbf{B}$ and $\mathbf{C c}$ by the other.

$$
\mathrm{X} \underset{k_{-1}}{\stackrel{k_{1}}{\rightleftharpoons}} \mathrm{Y} \underset{k_{-2}}{\stackrel{k_{2}}{\rightleftarrows}} \mathrm{Z}
$$


Applying the steady state hypothesis to Y, eq.(10) can be deduced. ${ }^{7}$

$$
k_{\text {bell }}=\frac{\frac{\left[H^{+}\right]}{\left[H^{+}\right]+K_{a}} K_{h} K_{t} K_{i}+k_{i}\left[H^{+}\right]}{\left[H^{+}\right]+\frac{k_{i} K_{t}}{k_{h}}}
$$

Eq.(10) gives a bell-shaped curve when represented as a function of pH. In Fig. 3, the bell shaped curve of luteolinidin, Scheme 1, is shown.

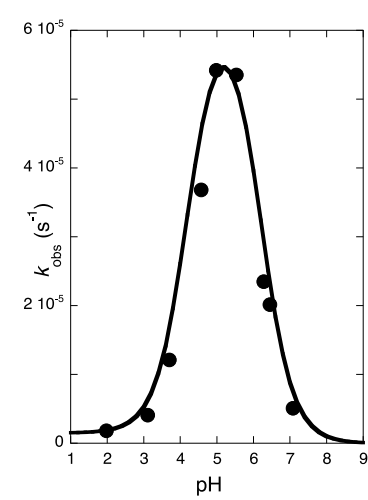

(a)

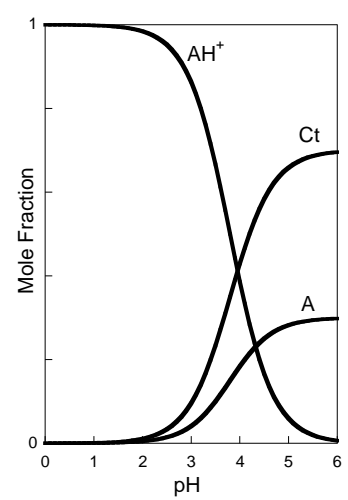

(b)

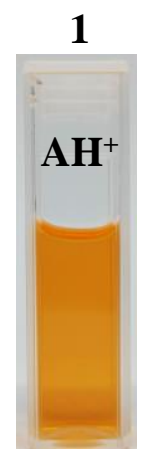

(c)

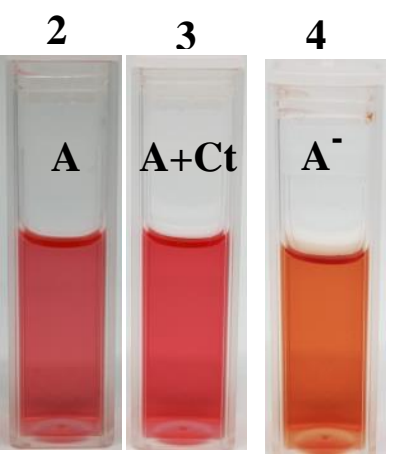

Figure 3. (a) Bell-shaped curve of the reaction towards the equilibrium versus $\mathrm{pH}$ of luteolinidin $2.5 \times 10^{-5} \mathrm{M}$ in ethanol:water (1:1). Fitting was achieved by means of eq.(10) for $K_{\mathrm{h}} K_{\mathrm{t}} K_{\mathrm{i}}=4 \times 10^{-9} \mathrm{M}^{-1} \mathrm{~s}^{-1}$; $K_{t} k_{i} / k_{h}=6.5 \times 10^{-7} \mathrm{M}^{-1} \mathrm{~s}^{-1} ; k_{-\mathrm{i}}=1.5 \times 10^{-6} \mathrm{~s}^{-1} ; \mathrm{p} K_{\mathrm{a}}=3.8$; (b) Mole fraction distribution of the species at the equilibrium, which is basically constituted by quinoidal base in equilibrium with trans-chalcone; (c) (1) Flavylium cation at $\mathrm{pH}=1.0$, (2) quinoidal base immediately after a $\mathrm{pH}$ jump to $\mathrm{pH}=5$; (3) at the equilibrium at $\mathrm{pH}=5$; (4) immediately after a $\mathrm{pH}$ jump to $\mathrm{pH}=8.0$. Immediately after a direct $\mathrm{pH}$ jump to $\mathrm{pH}=12$ the colour is magenta (not shown).

It is worth noting for the following discussion that the flavylium cation of luteolinidin is yellow/orange and the quinoidal base is red. At the equilibrium the colour is given by a mixture of $\mathbf{C t}$ (major species) and the red quinoidal base (minor species). This colour distribution is very different from the one of anthocyanins, Fig. 2c compared with Fig. 3c. Moreover, $\mathbf{B}$ and $\mathbf{C c}$ are elusive species, observed during the kinetic studies but not present at the equilibrium. On the other hand, the ionized quinoidal base is dark orange. It is a matter of fact that no blue colour can be achieved with luteolinidin, even at more basic $\mathrm{pH}$ values. 


\section{Photoinduced cis-trans isomerization}

One of the most interesting properties of the multistate is its photoinduced trans-cis isomerization, exemplified in Figure 4 for the model compound 4'-methoxyflavylium. ${ }^{10}$

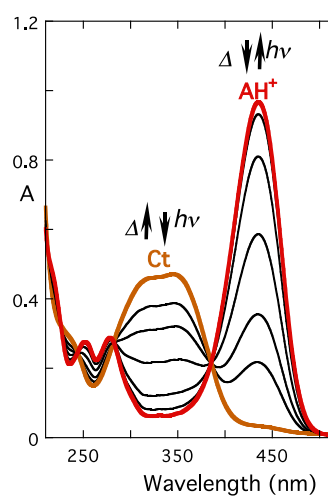

(a)

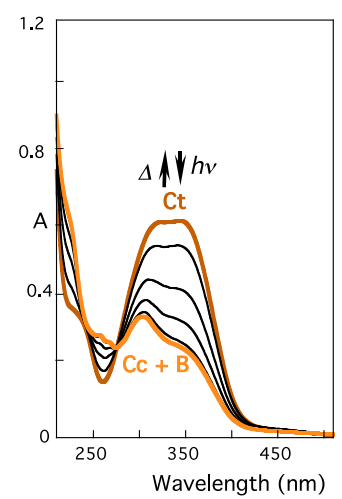

(b)

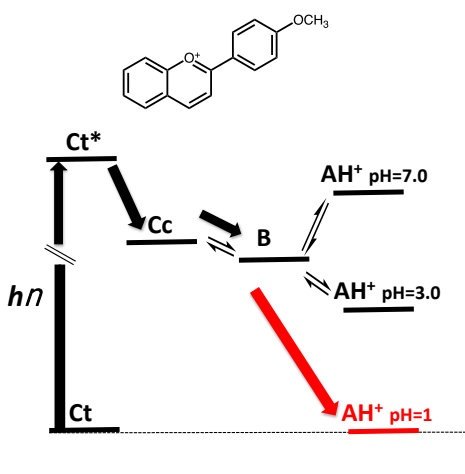

(c)

Figure 4. (a) $\mathrm{pH} 1.0,[\mathrm{Ct}]=2.5 \times 10^{-5} \mathrm{M}$; the curves correspond to the irradiation times $0.0 .5,1,2,4,7$ and $12 \mathrm{~min}$; $\lambda_{\mathrm{exc}}=365 \mathrm{~nm}$; (b) Spectral variations of 4'-methoxyflavylium at $\mathrm{pH}=7.0,[\mathrm{Ct}]=3.2 \times 10^{-5} \mathrm{M}$; the curves correspond to the irradiation times $0,0.25,1.5,3,6$, and $10 \mathrm{~min} ; \lambda_{\mathrm{exc}}=365 \mathrm{~nm}$; (c) Energy level diagram of 4'-methoxyflavylium. Adapted from reference [10].

The compound 4'-methoxyflavylium possesses a very high cis-trans isomerization barrier and by consequence $\mathbf{C t}$, the stable species at higher $\mathrm{pH}$ values, is also metastable at $\mathrm{pH}=1.0$. Consequently, irradiation of $\mathbf{C t}$ at $\mathrm{pH}=1.0$ leads to $\mathbf{C c}$ that gives $\mathbf{B}\left(k_{\mathrm{obs}}=0.95 \mathrm{~s}^{-1}\right)$ and this one produces flavylium cation $\left(k_{\mathrm{obs}}=14 \mathrm{~s}^{-1}\right)$, Fig. $4 \mathrm{a}$. In this case, there is no reversibility. Conversely, at $\mathrm{pH}=7.0$ the flavylium cation is not thermodynamically accessed, Fig. 4c, and the photoproducts are a mixture of $\mathbf{C c}$ and B $\left(K_{\mathrm{t}}=0.5\right)$, Fig. $4 \mathrm{~b}$. Reversibility back to $\mathbf{C t}$ is observed.

In anthocyanins, the mole fraction distribution of $\mathbf{C t}$ at the equilibrium is small and by consequence their photochromic systems are poor. However, in 3deoxyanthocyanidins $\mathbf{C t}$ is the major species and efficient photochromic systems have been described. ${ }^{13}$ 


\section{Results and Discussion}

Furanoflavylium molecules have long been prepared and identified as anthocyanins and related compounds, Scheme 3. ${ }^{14}$ Some of these molecules are natural products such as Riccionidin A, identified in several liverworts as for example in the Antarctic Cephaloziella varians, in response to an abrupt increase of UVB radiation, ${ }^{15}$ in vitro cultures of Ricciocarpos natans, ${ }^{16}$ as well as in adventitious root cultures of the anacardiaceae Rhus Javanica. ${ }^{17}$

Related to this research, the kinetics and thermodynamics of riccionidin A model compounds 4',7-dihydroxyfuranoflavylium and 4'-hydroxyfuranoflavylium were reported. ${ }^{8,9}$ It was proved that these auronidin model compounds follow the same multistate of species of anthocyanins where aurones are the trans-chalcones of the respective furanoflavylium salts, Scheme $2 .^{8}$

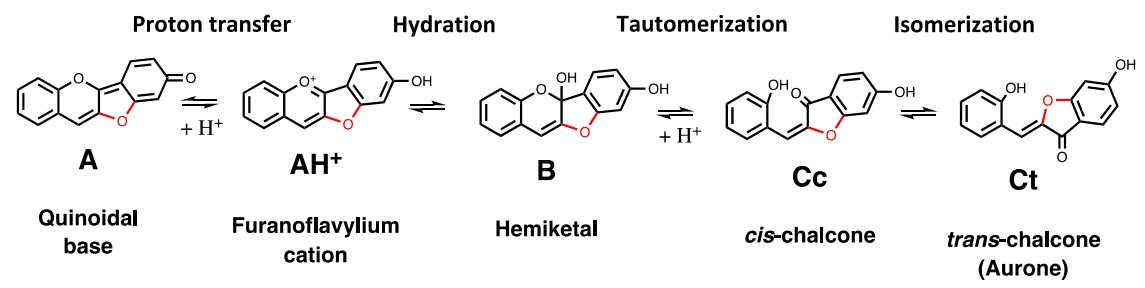

Scheme 2. Multistate of the chemical species of 4'-hydroxyfuranoflavylium in acid to moderately acidic medium. ${ }^{8}$

In spite of the similarities of furanoflavylium multistates with those of anthocyanins and deoxyanthocyanins, the rates for interconversion of the several species in auronidin model compounds are dramatically slower ${ }^{8,9}$ It is noteworthy the fact that the auronidin model compounds in Scheme 1 are better photoacids than the equivalent models of anthocyanins (without the furan bridge between rings $\mathrm{B}$ and $\mathrm{C}$ ). ${ }^{9}$ This means that furanoflavylium compounds are very appropriate molecules to dissipate by excited state proton transfer the electronic energy acquired upon light absorption.

The synthesis of riccionidin A was reported by Dyker and Bauer. ${ }^{18}$ The suitable aldehyde and ketone were dissolved in acetic acid saturated with $\mathrm{HCl}$ gas at $100{ }^{\circ} \mathrm{C}$, to give initially the trans-chalcone, see experimental section for more details. The evolution of the synthesis was monitored taking an aliquot of $20 \mu \mathrm{l}$ from the reaction vessel and dilute with $3 \mathrm{ml}$ of 1:1 (v/v) methanol: $\mathrm{HCl}(0.2 \mathrm{M})$ solution, Fig. 5a. This result confirms the extremely slow conversion of the trans-chalcone into flavylium 
cation, a result that was already observed by ${ }^{1} \mathrm{H}$ NMR and UV-vis absorption in model compounds of riccionidin A (furanoflavylium compounds), Scheme 1.,9

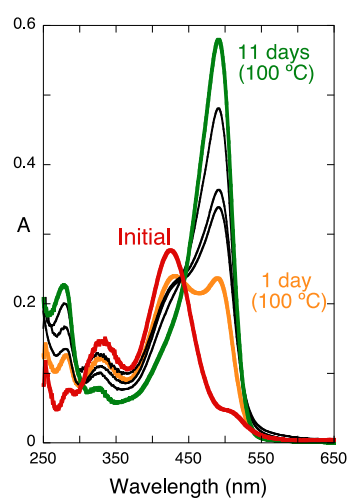

(a)

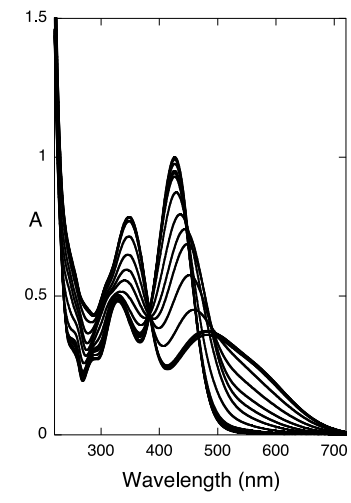

(b)

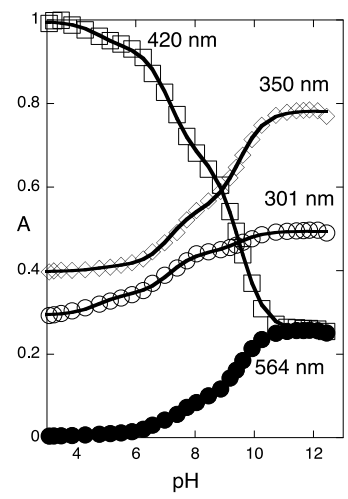

(c)

Figure 5. (a) Spectral variations during the synthesis of riccionidin A; (b) Spectral variations of transchalcone (immediately after the $\mathrm{pH}$ jump) as a function of $\mathrm{pH},[\mathrm{Ct}]=5.8 \times 10^{-5} \mathrm{M}$; (c) Respective titration curves.

As shown in Fig. 5a the first spectrum (red spectrum) exhibits the characteristic absorption band of trans-chalcone and even at high temperature and very acidic conditions the flavylium cation is formed (green coloured) very slowly. The ${ }^{1} \mathrm{H}$ NMR of both species, trans-chalcone and flavylium cation, are in accordance to those reported in literature. ${ }^{18}$

The spectral variations of the trans-chalcone upon purification by column chromatography and taken immediately after direct $\mathrm{pH}$ jumps, Fig. 5b and Fig. 5c, are compatible with the existence of three acid-base equilibria tentatively identified in the scheme 3 .

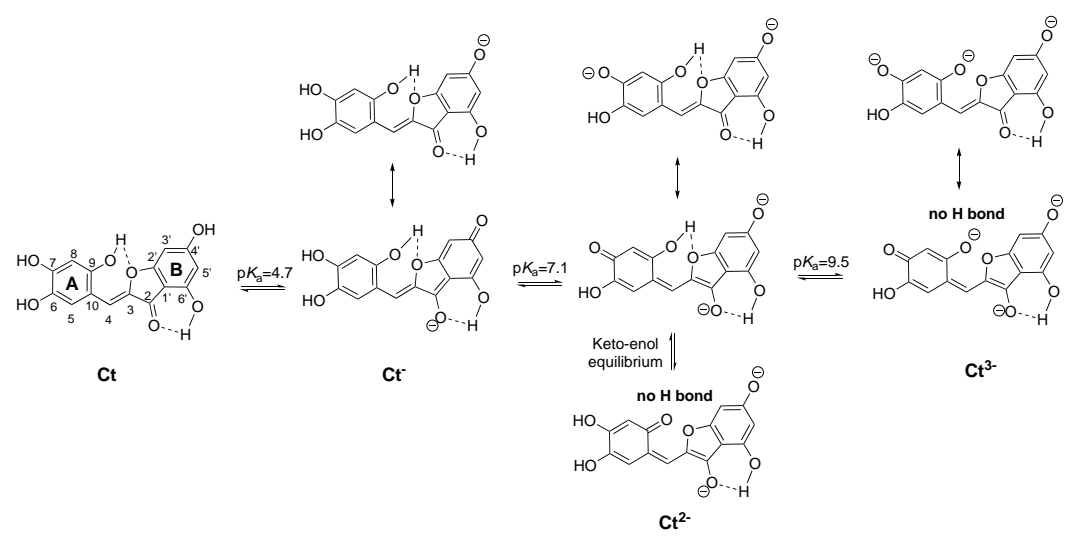

Scheme 3. Proposed protonation sequence for the trans-chalcone species. The numeration of the flavylium cation was used for comparison purposes. 
The spectral variations during the trans-chalcones reaction at different $\mathrm{pH}$ values is shown in Fig. 6. In particular, at $\mathrm{pH}$ values in the interval $5<\mathrm{pH}<7.5$, a new species exhibiting a blue-shifted absorption bands, is formed, Fig. 6a. For longer reaction times the isosbestic points are lost indicating formation of other products not belonging to the multistate, below identified as decomposition products. The $\mathrm{pH}$ dependence of the rate constants before the appearance of these products versus $\mathrm{pH}$ can be interpreted considering a fitting obtained through eq.(11)

$k_{o b s}=a_{c t}+b_{c t}+c_{c t 2}+d_{c t 3}$

where represents the mole fractions of the trans-chalcones in the different protonation states and the coefficients $a, b, c, d$ the respective rate constants respectively, $a \approx b=4.4 \times 10^{-6} \mathrm{~s}^{-1}, c=2.7 \times 10^{-3} \mathrm{~s}^{-1}$ and $d=5.0 \times 10^{-3} \mathrm{~s}^{-1}$.

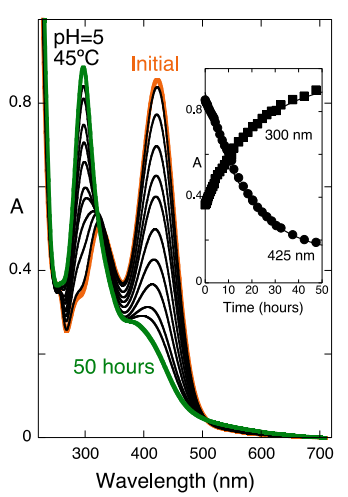

(a)

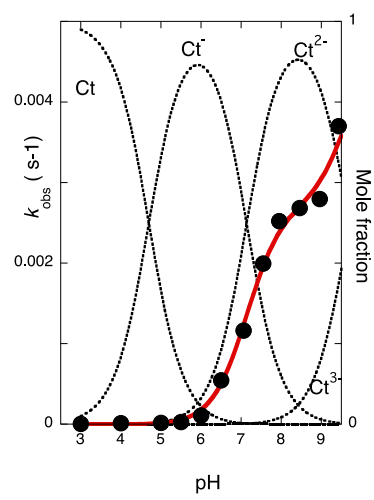

(b)

Figure 6. (a) Spectral variations of the trans-chalcone at $\mathrm{pH}=5.0$ in methanol:water $(1: 1)$ at $45{ }^{\circ} \mathrm{C}$, $[\mathrm{Ct}]=5.0 \times 10^{-5} \mathrm{M}$. The same behavior is observed in the $\mathrm{pH}$ range $1<\mathrm{pH}<6$; (b) Variation of the observed rate constant as a function of $\mathrm{pH}$; fitting was achieved for $\mathrm{a} \approx b=4.4 \times 10^{-6} \mathrm{~s}^{-1}, \mathrm{c}=2.7 \times 10^{-3} \mathrm{~s}^{-1}$ and $\mathrm{d}=5.0 \times 10^{-3} \mathrm{~s}^{-1}$. The last value is a rough estimation due to the decomposition process associated.

Considering that the first reaction towards formation of other products of the multistate is the isomerization of the chalcones, the rate dependence on the protonation state of the trans-chalcone can be explained with the help of Scheme 3. Ct species could be able to form up to two different intramolecular hydrogen bonds which confer an additional stability to the structure and difficult the cis-trans isomerization. Similarly, $\mathbf{C t}^{-}$species can establish a hydrogen bond which also slows down the isomerization process. Differently is the case of $\mathbf{C t}^{2-}$ and $\mathbf{C t}^{\mathbf{3 -}}$ due to the 
ionization of the A ring which promotes the breakdown of this last hydrogen bond and therefore facilitates the cis-trans isomerization.

More information regarding the riccionidin A multistate was obtained by HPLC-MS. The chromatographic profile as a function of time for the trans-chalcone form of riccionidin $\mathrm{A}$ is shown in Fig. 7 (0.5 mM at pH=7.1, in methanol:water (1:1), at room temperature). Peak 4 corresponds to the initial trans-chalcone. The $\mathrm{m} / \mathrm{z}$ value of peaks 3 and 7 and the correspondent absorption spectra observed in HPLC-DAD are compatible with two other species of the multistate, respectively, hemiketal and cischalcone. Differently, neither peak 1 ( $\mathrm{m} / \mathrm{z}=319$, negative mode), peak $2(\mathrm{~m} / \mathrm{z}=337$, negative mode), peak $5(\mathrm{~m} / \mathrm{z}=154$, negative mode) nor peak $6(\mathrm{~m} / \mathrm{z}=617$, negative mode) are compatible with any expected component of the multistate. A proposed mechanism with structures compatible with these MS results is reported in Scheme 4.

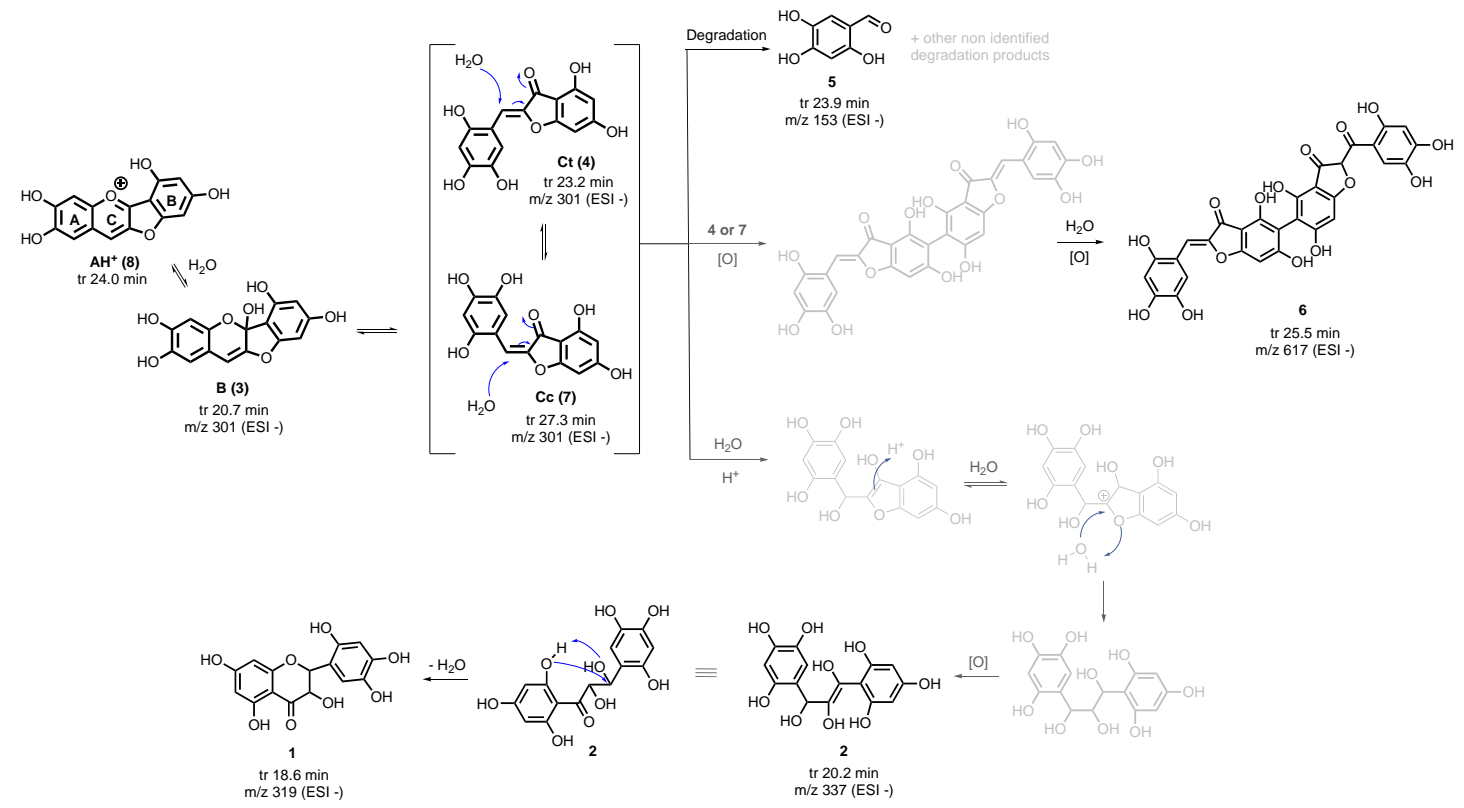

Scheme 4. Proposed chemical structures for peaks 1, 2, 5 and 6 detected by HPLC-MS and possible mechanistic pathways leading to their formation. The position of the methyl group was randomly assigned in the structure from peak 6.

The absorption spectra of the chromatographic peaks shown in Fig.7b contribute to the identification of the several chromatographic peaks. While peak 4 is unequivocally trans-chalcone, peak 7 is compatible with cis-chalcone: $i$ ) it is the first peak to appear after disappearance of peak 4, ii) the shape and position of the respective absorption spectra is the one expected for the cis-chalcone (slightly redshifted and less intense when compared with $\mathbf{C t}$ ). On the other hand, the absorption 
spectrum of peak 3, with a strong absorption in the UV, is compatible with the unconjugated hemiketal B. Peak 2, also with strong absorption in the UV, is compatible with a structure where conjugation is interrupted by a $\mathrm{C} \mathrm{sp}^{3}$ carbon, like in B. The fact that the peaks of B and Cc are well separated by HPLC indicates that the tautomerization reaction is also slower than in anthocyanins and 3-deoxyanthocyanins.

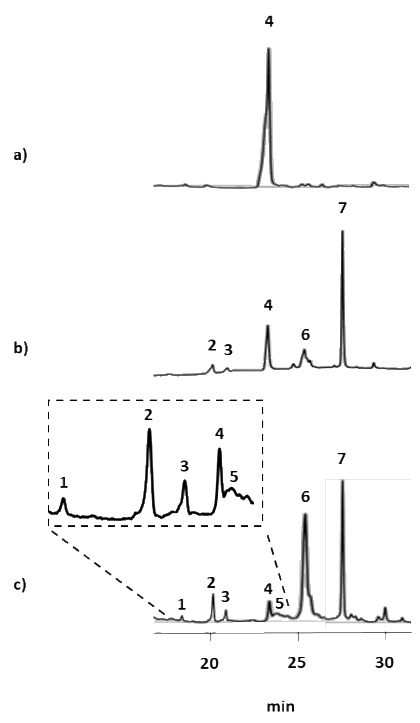

(a)

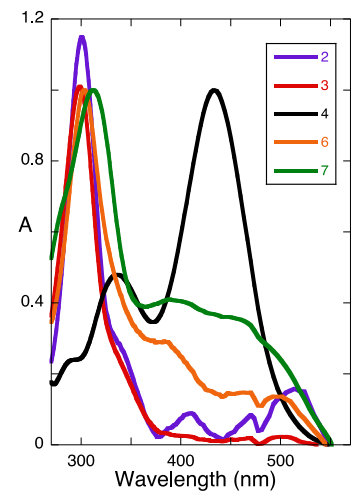

(b)

Figure 7. (a) Chromatographic profile of trans-chalcone (4) in methanol:water $1: 1(0.5 \mathrm{mM}, \mathrm{pH}=7.1)$ at $300 \mathrm{~nm}$. a) freshly prepared; (b) after 4 days at r.t.; c) after 10 days at r.t. $\mathrm{m} / \mathrm{z}=301$ (negative mode) for peaks 3, 4 and 7; m/z=319 (negative mode) for peak $1 ; \mathrm{m} / \mathrm{z}=337$ (negative mode) for peak 2; $\mathrm{m} / \mathrm{z}=154$ (negative mode) for peak $5 ; \mathrm{m} / \mathrm{z}=617$ (negative mode) peak 6 .

The photochemical reaction is another source of information for the system. In Fig. $8 \mathrm{a}$, the spectral variations upon irradiation of $\mathbf{C t}$ in acetonitrile containing a drop of trifluoroacetic acid are shown. The spectral variations shown in Fig. 8a clearly indicate the disappearance of $\mathbf{C t}$. The HPLC chromatograms taken at different irradiation times is presented are Fig. 8b. The initial process is, as expected, formation of cis-chalcone upon photoisomerization of $\mathrm{Ct}$, accounting for the appearance of peak 7. The formation of peak 3 (hemiketal) from cis-chalcone can take place thermally or upon excitation of cis-chalcone via excited state proton transfer from $\mathrm{Cc}^{*}$ to $\mathrm{B}^{*}$ (tautomers). Peak 2 grows with 3 suggesting that structure 2 derives from 3 as proposed in Scheme 4. 


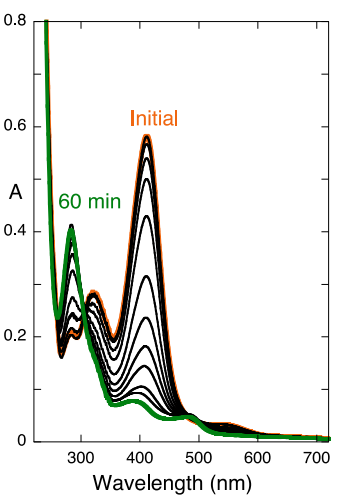

(a)

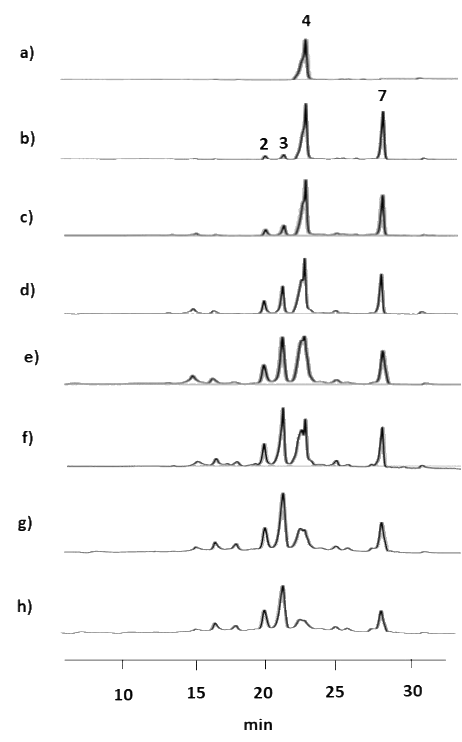

(b)

Figure 8. (a) Irradiation of trans-chalcone at $366 \mathrm{~nm}$ in acetonitrile (with a drop of TFA); $[\mathrm{Ct}]=3.4$ $\mathrm{x} 10^{-5} \mathrm{M}$; (b) Chromatographic profile of trans-chalcone solution (4) in acetonitrile $(0.66 \mathrm{mM})$ at $300 \mathrm{~nm}$. a) before irradiation; b) after $6 \mathrm{~min}$ of irradiation $(366 \mathrm{~nm})$; c) after $16 \mathrm{~min}$ of irradiation (366 $\mathrm{nm})$; d) after $35 \mathrm{~min}$ of irradiation (366 nm); e) after $1 \mathrm{~h}$ of irradiation (366 nm); f) after $1 \mathrm{~h}$ and $35 \mathrm{~min}$ of irradiation $(366 \mathrm{~nm}) ; \mathrm{f}$ ) after $2 \mathrm{~h}$ and $40 \mathrm{~min}$ of irradiation $(366 \mathrm{~nm})$; g) after $3 \mathrm{~h}$ and $40 \mathrm{~min}$ of irradiation $(366 \mathrm{~nm})$.

Flavylium cation was also purified by column chromatography and a set of direct $\mathrm{pH}$ jumps were carried out to an extended range of higher $\mathrm{pH}$ values. The respective kinetic processes are very slow in all $\mathrm{pH}$ ranges and after a few hours no significant spectral variations were detected. However, depending on $\mathrm{pH}$, spectral variations are observed after several days, see below. The $\mathrm{pH}$ dependent absorption spectra taken immediately after the direct $\mathrm{pH}$ jumps are shown in Fig. 9 and are compatible with a tetra-protonated acid, exhibiting well defined isosbestic points in four $\mathrm{pH}$ intervals.

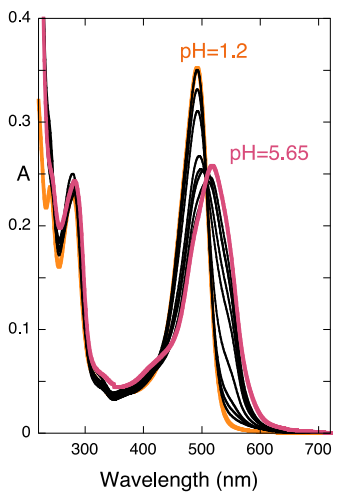

(a)

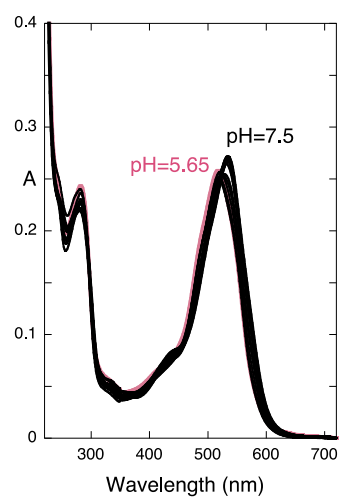

(b)

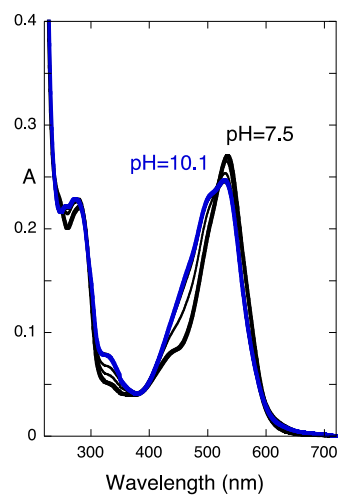

(c)

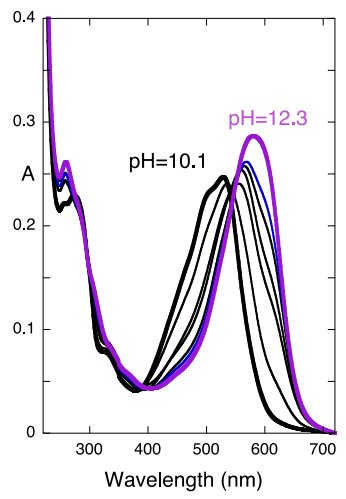

(d) 
Figure 9. $\mathrm{pH}$ dependent spectral modifications of riccionidin $\mathrm{A},\left[\mathrm{AH}^{+}\right]=3.3 \times 10^{-5} \mathrm{M}$ upon $\mathrm{pH}$ jumps from $\mathrm{pH}=1.0$ to higher $\mathrm{pH}$ values in the following $\mathrm{pH}$ intervals: (a) $1.2<\mathrm{pH}<5.65$; (b) $5.65<\mathrm{pH}<7.5$; (c) $7.5<\mathrm{pH}<10.1$; (d) $10.1<\mathrm{pH}<12.3$.

This result was confirmed by the titration curves reported in Fig. 10a. Four inflection points were identified allowing determination of four $\mathrm{p} K_{\mathrm{a}}$ values as expected from the four hydroxyl substituents of riccionidin A structure, Scheme 1.

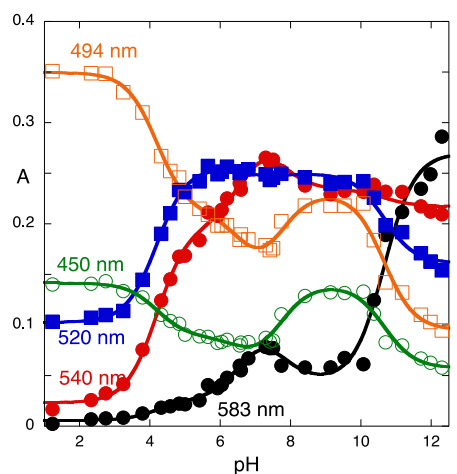

(a)

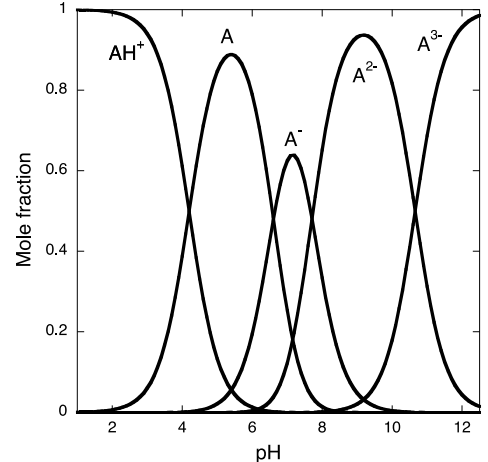

(b)

Figure 10. (a) Spectral variations of riccionidin A taken immediately after direct $\mathrm{pH}$ jumps from the flavylium cation equilibrated at $\mathrm{pH}=1.0$. The system behaves as a tetra-protic acid with $\mathrm{p} K_{\mathrm{a} 1}=4.2$; $\mathrm{p} K_{\mathrm{a} 2}=6.6 ; \mathrm{p} K_{\mathrm{a} 3}=7.7 ; \mathrm{p} K_{\mathrm{a} 4}=10.7 ;(\mathbf{b}) \mathrm{pH}$ dependent mole fraction distribution of the several species.

Once calculated the acidity constants, the mole fraction distribution of the several species that result from the deprotonation of the phenol groups of the flavylium cation is straightforwardly obtained, Fig.10b. A tentative protonation sequence is shown in Scheme 5, using as starting point the deprotonation sequence observed in the model compound 4,7-dihydroxyfuranoflavylium. ${ }^{9}$

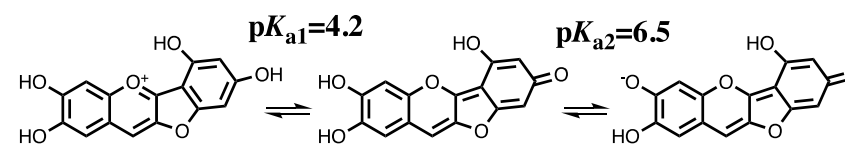

$\mathrm{AH}^{+}$

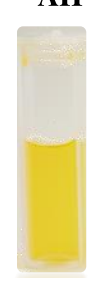

pH 1.0
A

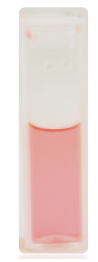

pH 5.5
$\mathbf{A}^{-}$

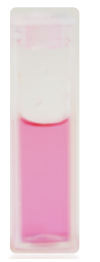

pH 7.3

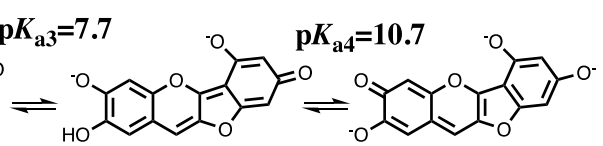

$\mathrm{A}^{2-}$

$\mathrm{A}^{3-}$

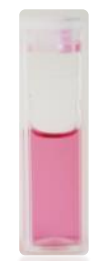

pH 9.0

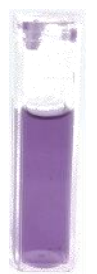

pH 12.0

Scheme 5. Top: proposed deprotonation sequence for flavylium cation of riccionidin A; bottom: colour of the flavylium cation and quinoidal bases of riccionidin $\mathbf{A}$ immediately after the respective preparation. Only at extremely basic $\mathrm{pH}$ values a bluish-purple colour is observed. No blue colour like those of ionized quinoidal bases in anthocyanins was achieved. 
Relevant information of the system regards the colour of the quinoidal base and flavylium cation, Scheme 5, down. Similarly to the natural auronidin ${ }^{1}$ the red colour observed in riccionidin $\mathrm{A}$ is given by the quinoidal base.

Evolution of the initial absorption spectra reported in Fig. 9 is shown in Fig. 11. No significant spectral modifications were observed after 500 hours for $\mathrm{pH}<4$, indicating that the flavylium cation is stable in that $\mathrm{pH}$ region. Fig. 11a, $11 \mathrm{~b}$ and $11 \mathrm{c}$ show the spectral variations at three representative $\mathrm{pH}$ values where the species $\mathrm{A}, \mathrm{A}^{-}$and $\mathrm{A}^{2-}$ are the major ones at zero time. In the three cases the kinetics are bi-exponential. The $\mathrm{pH}$ jump to $\mathrm{pH}=9.1$ is extremely slow and some decomposition takes place.

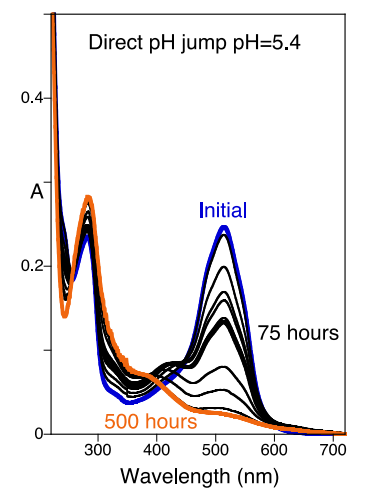

(a)

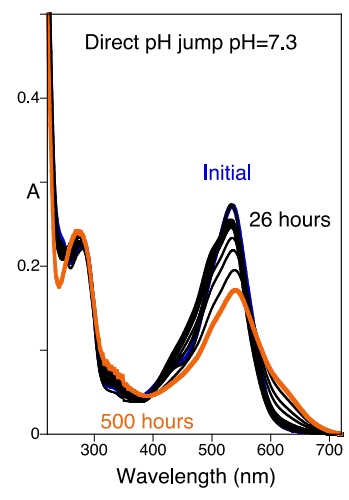

(b)

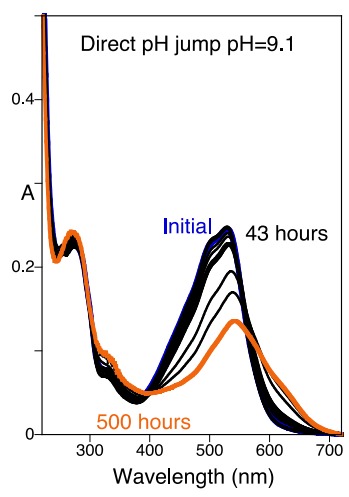

(c)

Figure 11. Kinetics of the direct $\mathrm{pH}$ jumps from flavylium cation at $\mathrm{pH}=1.0$ at room temperature, $\left[\mathrm{AH}^{+}\right]=3.3 \times 10^{-5} \mathrm{M}$.

At higher $\mathrm{pH}$ values the absorption spectra of the quinoidal bases is still observed. At $\mathrm{pH}=5.4$ the absorption spectrum after 500 hours resembles those previously reported for peak 6 (see Scheme 4) in Fig. 7.

The reaction kinetics of flavylium cation solutions after a direct $\mathrm{pH}$ jump from $\mathrm{pH}=1.0$ to $\mathrm{pH}=5.8$ (taken at $45^{\circ} \mathrm{C}$ to decrease the reaction times), were followed by HPLC, Fig. 12. The mobile liquid phase of the elution gradient varies between $1<\mathrm{pH}<2$. Consequently, all quinoidal bases are transformed in flavylium cation. The HPLC profile after 10 minutes already shows a peak (peak 7) that we attributed to cischalcone. After 24 hours the cis-chalcone peak starts to decrease and peak 6 appears. The absorption spectrum of this peak is coincident with the one of Fig. 6a after 500 hours and Fig. 7b. 


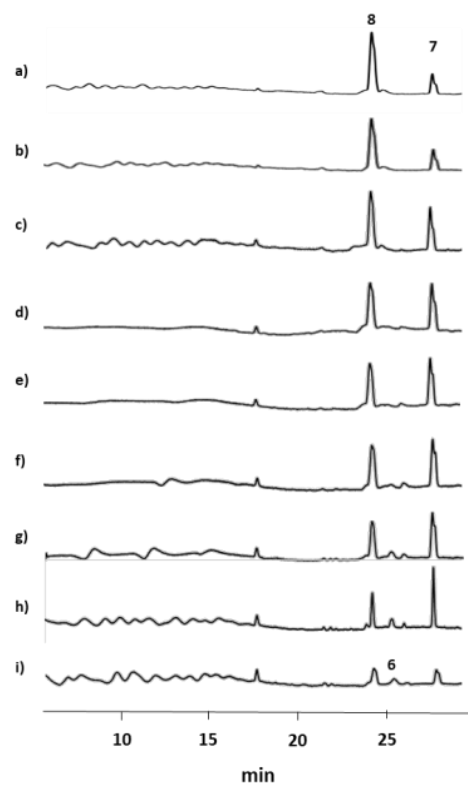

Figure 12. Chromatographic profile (at $300 \mathrm{~nm}$ ) of flavylium salt (compound 8) solution (methanol:water $1: 1 ; 1.15 \mathrm{mM}$ ) after a $\mathrm{pH}$ jump to $\mathrm{pH}=5.8$ and at $45^{\circ} \mathrm{C}$. a) after $10 \mathrm{~min}$.; b) after 20 min.; c) after 1 h.; d) after 2 h.; e) after 4 h.; f) after 8 h.; g) after 24 h.; h) after 33 h.; c) after 48 h.

The data from Fig. 12 shows also that after $48 \mathrm{~h}$ the formation of more decomposition products are very significant. This prevents the definition of the equilibrium.

\section{Conclusions}

The plants where the three multistates have been found, respectively liverworts (auronidins), mosses and ferns (deoxyanthocyanins) and, angiosperms (anthocyanins) are in different positions in the evolution tree. The former are the older and the latter the newer. In the case of the multistate of the natural compound riccionidin A described through this work, as well as its synthetic model compounds, the most interesting feature is the extremely low rate of the interconversion between the several species of the multistate. This constitutes a handicap for the production of red colour by the plant in response to external stimuli. 3-Deoxyanthocyanidins multistate interconnect much faster than auronidins but still have a slower response when compared with anthocyanins. Neither auronidins nor deoxyanthocyanins multistates have species with blue colour, even in basic medium. Anthocyanins are more versatile in the colour pallet and the interconnection between the multistate species is much faster, permitting to respond more efficiently to natural external stimuli such as $\mathrm{pH}$ and light. This study complements from a chemical point of view the studies 
developed from evolutionary plant biology and opens perspectives in the design and characterization of new pigments characterized by a high stability over an extended $\mathrm{pH}$ range when compared to anthocyanins.

\section{Experimental Section}

Materials and methods. All solvents and chemicals employed in syntheses and preparation of samples were reagent or spectrophotometric grade and were used as received; Millipore-grade water was used. NMR spectra were run on a Bruker Advance III 400 spectrometer (400 MHz for ${ }^{1} \mathrm{H}, 100 \mathrm{MHz}$ for ${ }^{13} \mathrm{C}$ ) at $298 \mathrm{~K}$. Highperformance liquid chromatography (HPLC) analyses were conducted on a MerckHitachi instrument equipped with a diode array detector (DAD), scan range 200-800 nm (Merck-Hitachi L-4500 Diode Array Detector), operating at $25{ }^{\circ} \mathrm{C}$, using a reversed-phase analytical column (RP-HPLC, Purosphere® Star column, $250 \mathrm{~mm} \times 3$ mm i.d., $5 \mu \mathrm{m}$ ). Samples were prepared in $\mathrm{MeOH}$ and the injection volume was 20 $\mu \mathrm{L}$. The best peak separation was obtained with $\mathrm{H}_{2} \mathrm{O}: \mathrm{HClO}_{4}, 99.7: 0.3$, v/v (solvent A) and pure $\mathrm{MeOH}$ (solvent $\mathrm{B}$ ) at a flow rate of $1 \mathrm{~mL} / \mathrm{min}$ : 7\% $\mathrm{B}$ for 2 min; linear gradient from $7 \%$ to $15 \% \mathrm{~B}$ for $6 \mathrm{~min}$; linear gradient from $15 \%$ to $75 \% \mathrm{~B}$ for $17 \mathrm{~min}$; linear gradient from $75 \%$ to $80 \%$ B for 2 min; linear gradient from $80 \%$ to $100 \%$ B for another $2 \mathrm{~min} ; 100 \% \mathrm{~B}$ for $11 \mathrm{~min}$ and $5 \mathrm{~min}$ to return to the initial condition. The total run time excluding equilibration was $40 \mathrm{~min}$. HPLC-MS analyses were performed in an Agilent 1200 Series equipment coupled with an Agilent 6130B Single Quadrupole detector with API-ES source. Column chromatography was carried out using Macherey-Nagel Polygoprep® 300-50C18. The column was packed with a mixture of stationary phase and $\mathrm{H}_{2} \mathrm{O}: \mathrm{MeOH} 80: 20 \mathrm{v} / \mathrm{v}(\mathrm{pH}=1.0)$. Gradient elution of increased $\mathrm{MeOH}$ proportion was performed to achieve separation. Separation was followed by HPLC and by fraction's weight.

Thermodynamic and kinetic studies. The $\mathrm{pH}$ jumps were carried out by adding a stock solution of flavylium salt or trans-chalcone in $\mathrm{HCl} 0.1 \mathrm{M}(1 \mathrm{~mL})$ to a $3 \mathrm{~mL}$ quartz cuvette containing a solution of $\mathrm{NaOH} 0.1 \mathrm{M}(1 \mathrm{~mL})$ and universal buffer of Theorell and Stenhagen $(1 \mathrm{~mL})^{19}$ at the desired final $\mathrm{pH}$. This defined the ionic strength as 0.1 $\mathrm{M}$ (controlled by the $\mathrm{NaCl}$ concentration resulting from neutralization). The final $\mathrm{pH}$ of the solutions was measured in a Crison basic $20+\mathrm{pH}$ meter. Spectroscopic measurements were performed using Mili-Q water with a constant temperature of $20 \pm 1^{\circ} \mathrm{C}$, with a Varian-Cary 100 Bio spectrophotometer. 
Synthesis of (Z)-4,6-dihydroxy-2-(2,4,5-trihydroxybenzylidene)benzofuran-3(2H)-one (trans-chalcone of Riccionidin A) (3). The synthesis of compound $\mathbf{3}$ was achieved according to the experimental procedure previously described with some minor modifications. ${ }^{20}$ Through a solution of $23 \mathrm{mg} \quad(0.15 \mathrm{mmol})$ of 2,4,5trihydroxybenzaldehyde (previously prepared according to literature) ${ }^{21}$ and $26 \mathrm{mg}$ (0.16 mmol) of 4,6-dihydroxybenzofuran-3(2H)-one (previously prepared according to literature $)^{22}$ dissolved in $2 \mathrm{ml}$ of acetic acid, dry hydrogen chloride was bubbled during $45 \mathrm{~min}$ at room temperature. After $24 \mathrm{~h}$ of additional stirring the mixture was purified by RP-column chromatography, yielding pure compound 3 ( $21 \mathrm{mg}, 0.07$ mmol, 50\%). NMR data of compound $\mathbf{3}$ are in accordance with those reported in literature. ${ }^{20}{ }^{1} \mathrm{H}$ NMR (400 MHz, MeOD- $\left.d_{4}\right) \delta 7.70(d, J=3.2 \mathrm{~Hz}, 1 \mathrm{H}), 7.22(d, J=$ $3.2 \mathrm{~Hz}, 1 \mathrm{H}), 6.41(s, 1 \mathrm{H}), 6.23(s, 1 \mathrm{H}), 6.05(s, 1 \mathrm{H}) .{ }^{13} \mathrm{C}$ NMR $\left(100 \mathrm{MHz}, \mathrm{MeOD}-d_{4}\right)$ $\delta 183.4,169.1,168.5,159.2,153.2$ (x2), 150.2, 147.4, 139.6, 118.5, 112.6, 108.1, 105.3, 104.4, 98.5, 92.0.

Synthesis of 2,3,6,8-tetrahydroxybenzofuro[3,2-b][1]benzopyrylium chloride (Riccionidin A; 2',4',6,7-tetrahydroxyfuranoflavylium chloride) (6). The synthesis of compound 6 was achieved according to the experimental procedure previously described with some minor modifications. ${ }^{20}$ Through a solution of $60 \mathrm{mg}(0.39 \mathrm{mmol})$ of 2,4,5- trihydroxybenzaldehyde (previously prepared according to literature ${ }^{21}$ and $65 \mathrm{mg}(0.39 \mathrm{mmol})$ of 4,6-dihydroxybenzofuran-3(2H)-one (previously prepared according to literature $)^{22}$ dissolved in $4 \mathrm{ml}$ of acetic acid, dry hydrogen chloride was bubbled during $45 \mathrm{~min}$ at room temperature. After 11 days of additional stirring at $100{ }^{\circ} \mathrm{C}$, the solution was allowed to cool to room temperature and $30 \mathrm{~mL}$ of $\mathrm{Et}_{2} \mathrm{O}$ were added leading to the appearance of a dark solid. The precipitate was filtered off and carefully washed with $\mathrm{Et}_{2} \mathrm{O}$ and dried, yielding flavylium salt 6 (0.103 g; 0.32 mmol; $82 \%$ yield). NMR data of compound $\mathbf{3}$ are in accordance with those reported in literature. ${ }^{20}{ }^{1} \mathrm{H}$ NMR (400 MHz, DMSO- $d_{6}$ :TFA 4:1) $\delta 8.99(s, 1 \mathrm{H}), 7.52(s, 2 \mathrm{H}), 6.66$ $(s, 1 \mathrm{H}), 6.54(s, 1 \mathrm{H})$. 


\section{Acknowledgments}

This work was supported by the Associate Laboratory for Green Chemistry -LAQV which is financed by national funds from FCT/MCTES (UIDB/50006/2020). FCT/MCTES is also acknowledged through the National Portuguese NMR Network RECI/BBB-BQB/0230/2012. A.A.-A. is grateful for the post-doctoral fellowship from Fundación Alfonso Martín Escudero. J. M. gratefully acknowledges a PhD grant from CONACyT (MEX/ Ref. 288188).

\section{References}

${ }^{1}$ H. Berland, N. W. Albert, A. Stavland, M. Jordheim, T. K. McGhie, Y. Zhou, H. Zhang, S. C. Deroles, K. E. Schwinn, B. R. Jordan, K. M. Davies, O. M. Andersen, PNAS, 2019, 116, 20232-20239.

${ }^{2}$ F. Pina, M. J. Melo, C. A. T. Laia, A. J. Parola, J. C. Lima, Chem. Soc. Rev., 2012, 41, 869-908.

${ }^{3}$ R. Brouillard, J.-E. Dubois, J. Am. Chem. Soc., 1977, 99, 1359-1364

${ }^{4}$ R. Brouillard, J. Lang, Can. J. Chem., 1990, 68, 755-761.

${ }^{5}$ R. A. McClelland, S. Gedge, J. Am. Chem. Soc., 1980, 102, 5838-5848.

${ }^{6}$ R. A. McClelland, G. H. McGall, J. Org. Chem., 1982, 47, 3730-3736.

7 F. Pina, J. Agric. Food Chem., 2014, 62, 6885-6897,

${ }^{8}$ A. Alejo-Armijo, A. J. Parola, F. Pina, ACS OMEGA, 2019, 3, 17853-17862.

${ }^{9}$ A. Alejo-Armijo, N. Basílio, A. Freitas, A. L. Maçanita, J. C. Lima, A. J. Parola, F. Pina, Physical Chemistry Chemical Physics, 2019, 21, 21651 - 21662.

${ }^{10}$ F. Pina, M. J. Melo, M. Maestri, R. Ballardini, V. Balzani, J. Am. Chem. Soc. 1997, 119, 5556-5561.

${ }^{11}$ A.L. Maçanita, P. F. Moreira, J. C. Lima. F. H. Quina, C. Yihwa, C. Voultier-Giongo J. Phys. Chem. A, 2002, 106, 1248-1255.

${ }^{12}$ F. Pina, A. J. Parola, M. J. Melo, J. C. Lima, V. de Freitas, chapter 2 in Anthocyanins from natural sources:exploiting targeted delivery for improved health, ed. M. S-L Brooks and G. B. Celli, Royal Society of Chemistry,U.K., 2019.

${ }^{13}$ M. J. Melo, S. Moura, A. Roque, M. Maestri, F. Pina, J. Photochem. Photobiology, 2000, 135, $33-$ 39.

${ }^{14}$ G. Chakravarty, T. R. Seshadri, Indian J. Chem. 1964, 2, 319-323.

${ }^{15}$ K. R. S. Snell, T. Kokubun, H. Griffiths, P. Convey, D. A. Hodgson, K. K. Newsham, Global Change Biology, 2009, 15, 2563-2573.

${ }^{16}$ S. Kunz, H. Becker, Z. Naturforsch, 1955, 50c, 235-240.

17 S. Taniguchi, K. Yazaki, R. Yabu-uchi, K. Kawakami, H. Ito, T. Hatano, T. Yoshida, Phytochemistry, 2000, 53, 357-363.

${ }^{18}$ G. Dyker, M. Bauer, J. Prakt. Chem., 1998, 340, 271-273.

${ }^{19}$ Küster, F. W.; Thiel, A.; Brückner, E. Tabelle per Le Analisi Chimiche e Chimico-fisiche. Hoepli: Milano, 1985; 157-160. The universal buffer used was prepared in the following way: $85 \%$ (w/w) phosphoric acid (2.3 mL), monohydrated citric acid (7.00 g), and boric acid (3.54 g) were dissolved in water; $1 \mathrm{M} \mathrm{NaOH}(343 \mathrm{~mL})$ was then added, and the solution was diluted to $1 \mathrm{~L}$ with water.

${ }^{20}$ G. Dyker, M. Bauer, J. prakt. Chem., 1998, 340, 271-273.

${ }^{21}$ Y. H. Seo, K. Damodar, J. Kim, J. Jun, Biorg. Med. Chem. Lett. 2016, 26, 1521-1524.

${ }^{22}$ A. Meguellati, A. Ahmed-Belkacem, A. Nurisso, W. Yi, R. Brillet, N. L. Chavoutier, A. Fortuné, J.M. Pawlotsky, A. Boumendjel, M. Peuchmaur, Eur. J. Med. Chem. 2016, 115, 217-229. 